\title{
Balkanologie
}

Balkanologie Revue d'études pluridisciplinaires

\section{Stabilisation et association dans les Balkans occidentaux : les défis du double objectif européen}

\section{Theresia Toeglhofer et Natasha Wunsch}

\section{(2) OpenEdition}

\section{Journals}

Édition électronique

URL : http://journals.openedition.org/balkanologie/2143

DOI : 10.4000/balkanologie.2143

ISSN : 1965-0582

Éditeur

Association française d'études sur les Balkans (Afebalk)

Référence électronique

Theresia Toeglhofer et Natasha Wunsch, « Stabilisation et association dans les Balkans occidentaux les défis du double objectif européen », Balkanologie [En ligne], Vol. XII, n 1 | 2010, mis en ligne le 15 février 2013, consulté le 17 décembre 2020. URL : http://journals.openedition.org/balkanologie/2143 ; DOI : https://doi.org/10.4000/balkanologie.2143

Ce document a été généré automatiquement le 17 décembre 2020.

(C) Tous droits réservés 


\title{
Stabilisation et association dans les Balkans occidentaux : les défis du double objectif européen
}

\author{
Theresia Toeglhofer et Natasha Wunsch
}

\section{NOTE DE L'AUTEUR}

Le manuscrit pour l'article a été terminé en décembre 2009.

\section{Introduction}

1 Après le 'big bang' de l'élargissement de l'Union européenne (UE) vers l'Europe centrale et orientale de 2004, complété en 2007 par l'adhésion de la Bulgarie et de la Roumanie, les Balkans occidentaux constituent désormais la prochaine région aux portes de l'UE. À la suite des violents conflits des années 1990, on a pu assister à une double évolution dans l'approche européenne vers la région : d'un côté, l'on constate une européanisation des sorties de crise dans le cadre du développement de la Politique européenne de sécurité et de défense (PESD) ${ }^{1}$ et, de l'autre, la transition d'une approche essentiellement sécuritaire vers un processus plus global, qui vise également des objectifs de développement et, à terme, l'intégration des Balkans occidentaux dans l'Union européenne'2.

2 C'est cette double orientation stratégique de l'UE, reflétée dans le Processus de stabilisation et d'association (PSA) conçu spécialement pour la région, qui se trouve au cœur de la présente analyse. Il s'agira d'abord de dresser un tableau des différentes facettes du PSA et de son évolution dans le temps pour en dégager l'orientation particulière qui résulte de la coexistence dans les Balkans occidentaux des transitions politique, économique et d'après-guerre. La mise en œuvre problématique du PSA, résultant de sa double dimension à la fois de stabilisation et d'association est ensuite 
analysée, avec une focalisation sur le rôle de la conditionnalité. Des études de cas de la Macédoine $^{3}$, de la Bosnie-Herzégovine et de la Serbie illustrent la tension entre considérations sécuritaires et exigences d'un strict exercice de la conditionnalité politique. L'article se conclut par une évaluation du PSA dans une perspective plus large, qui permet d'inclure d'autres facteurs pertinents pour sa mise en œuvre, tels que le rôle des dirigeants politiques dans la région et les difficultés à l'intérieur de l'Union.

\section{Les politiques européennes dans les Balkans occidentaux : entre endiguement et transformation}

3 La violente dissolution de la Yougoslavie au début des années 1990 posa un défi inattendu à la politique extérieure et de sécurité commune (PESC), que l'Union européenne s'apprêtait tout juste à incorporer dans sa structure institutionnelle comme deuxième pilier introduit par le Traité de Maastricht. Suite à la revendication célèbre de l'ancien Ministre des Affaires étrangères luxembourgeois, Jacques Poos, que l'heure de l'Europe était venue, l'impuissance de l'Union face à l'éclatement de la guerre ne fut que plus visible. Confrontée au déchirement sanglant de l'ancien État multinational, l'UE dut attendre l'intervention militaire de l'OTAN et la contribution décisive des États-Unis pour mettre fin à la violence - dans le cas de la BosnieHerzégovine, près de quatre ans après le début des hostilités.

4 Dans ce contexte, il semble évident que l'approche de l'UE en direction des Balkans occidentaux ne pouvait simplement suivre le chemin tracé par l'élargissement à l'Europe centrale et orientale. Aussi cette approche se caractérisa-t-elle tout d'abord par un souci de stabilisation post-conflit : en Bosnie-Herzégovine, les pays européens fournirent une grande partie des troupes de la force de stabilisation SFOR de l'ONU, qui demeura en place jusqu'au transfert de responsabilité à la mission européenne EUFOR Althea, en décembre 2004. Lors de l'opération de l'OTAN au Kosovo et en Serbie en 1999, plusieurs pays membres de l'Union furent également engagés aux côtés des ÉtatsUnis pour empêcher le nettoyage ethnique contre la population albanophone. Ces engagements militaires soulignent la précarité de la situation sécuritaire dans la région et la nécessité pour l'UE d'adopter une approche plus graduelle en vue du rapprochement des Balkans occidentaux à l'Europe.

5 Afin de discuter cette approche, il est nécessaire d'en saisir tout d'abord les caractéristiques essentielles, notamment celles contenues dans le processus de stabilisation et d'association. La conditionnalité renforcée pour les Balkans occidentaux est ensuite explicitée, pour enfin clore avec la question d'une possible transition de la stabilisation vers l'association.

\section{Le processus de stabilisation et d'association}

6 La perspective d'une association plus étroite avec l'UE, voire d'une intégration pleine et entière, fut mentionnée pour la première fois en juin 1999 lors du Conseil européen de Cologne dans le cadre du Pacte de stabilité pour l'Europe du Sud-Est. À cette occasion, l'UE annonça sa disposition «à rapprocher les pays de cette région de la perspective d'une pleine intégration dans ses structures $»^{4}$. Le Processus de stabilisation et d'association (PSA), formellement lancé au sommet Union européenne-Balkans occidentaux à Zagreb en novembre 2000, créa par la suite un partenariat progressif en 
vue d'une adhésion à terme à l'UE. Trois ans plus tard, par la Déclaration de Thessalonique de juin 2003 clôturant un nouveau sommet UE-Balkans Occidentaux, l'Union réaffirma "son soutien sans équivoque à la perspective européenne qui s'offre aux pays des Balkans occidentaux. L'avenir des Balkans est dans l'Union européenne ${ }^{5}$.

Le PSA repose sur trois grands piliers : la conclusion et la mise en œuvre d'un accord de stabilisation et d'association (ASA) individualisé avec chacun des pays de la région, incluant une libéralisation graduelle des échanges, une aide technique et financière (depuis 2007 à travers le nouvel instrument de pré-adhésion ou IPA, instrument unique se substituant à divers mécanismes précédents) ainsi que l'encouragement à la coopération régionale. Après un premier rapprochement sur le plan commercial et une assistance institutionnelle et financière pour déclencher le processus de réformes, l'ASA symbolise l'établissement d'une relation contractuelle entre l'Union européenne et chacun des candidats potentiels à l'adhésion.

Entre avril 2001 et juin 2008, tous les pays des Balkans occidentaux ont signé un tel accord de stabilisation et d'association ${ }^{6}$. Deux accords ne sont cependant pas encore entrés en vigueur : celui de la Bosnie-Herzégovine, qui était le dernier à être signé dans la région, attend encore sa ratification par plusieurs États membres de l'UE. Quant à la Serbie, elle fait face au refus des Pays-Bas d'avancer sur le chemin vers l'adhésion tant que les fugitifs Ratko Mladić et Goran Hadžić ne sont encore appréhendés et extradés vers le Tribunal pénal international de la Haye (TPIY). Bien que la mise en place de l'ASA serbe demeure suspendue, le rapport semestriel positif du procureur en chef du TPIY, Sergé Brammertz, publié en décembre 2009, a au moins permis le déblocage de l'accord intérimaire sur les dispositions commerciales de l'ASA'.

9 Dans le groupe des "candidats potentiels" constitué par la Bosnie-Herzégovine, la Serbie, le Monténégro et l'Albanie, les trois derniers ont récemment adressé une demande d'adhésion à l'UE. Tandis que le Monténégro est en train de remplir un questionnaire de la Commission portant sur sa capacité à incorporer l'acquis communautaire dans sa propre législation, la demande de l'Albanie a déjà été reçue par le Conseil, mais le pays attend encore la transmission du questionnaire. La Serbie vient tout juste de déposer sa demande le 22 décembre, et attend encore une réaction officielle de la part de l'UE'.

La Croatie et la Macédoine sont pour l'instant les candidats les plus avancés et les seules à avoir obtenu le statut officiel de candidat à l'adhésion, en juin 2004 pour Zagreb, suivie par Skopje en décembre 2005. Toutefois, en raison d'une dispute avec la Grèce portant sur son nom constitutionnel, la Macédoine attend encore l'ouverture des négociations d'adhésion. La Croatie négocie les chapitres de son traité d'adhésion depuis le 3 octobre 2005 et, à la suite de la résolution provisionnelle du conflit frontalier avec son voisin slovène, elle "s'approche de la dernière ligne droite $»^{9}$. Le processus d'association s'achèvera avec la clôture des négociations et, après l'avis de la Commission et l'approbation du Parlement et du Conseil européen, la signature du traité d'adhésion et sa ratification par le pays candidat et chaque Etat membre ${ }^{10}$.

\section{Une conditionnalité renforcée pour les Balkans occidentaux}

11 Comme pour les élargissements précédents, le principe de conditionnalité demeure au cœur du processus d'intégration. Ainsi, l'approche de l'Union se veut méritocratique, c'est-à-dire que l'avancement de chaque pays dépend de ses progrès accomplis dans la 
mise en œuvre des réformes. Liant l'association et, à terme, l'adhésion à l'Union européenne aux avancées dans l'agenda des réformes économiques et institutionnelles, la politique de conditionnalité s'est globalement révélée un levier de réformes efficace ${ }^{11}$. Cependant, comparé aux conditions requises lors des deux précédentes vagues d'adhésion, le contrôle des exigences vis-à-vis des Balkans occidentaux a été considérablement renforcé. Par ailleurs, étant donné l'état fragile des économies nationales, la situation politique partiellement instable et l'héritage d'un passé conflictuel récent, le Conseil européen du 29 avril 1997 a convenu d'autres conditions de caractère général s'ajoutant désormais aux critères de Copenhague définis en 1993. Intitulée 'principes fondamentaux' dans les accords,cette conditionnalité élargie concerne les réformes démocratiques (séparation des pouvoirs, indépendance des juges et des médias, code électoral), le respect des droits de l'homme et des minorités, le retour des réfugiés, les réformes économiques et la coopération régionale. Par ailleurs, il existe des conditions spécifiques à chaque pays fixées dans les ASA, portant notamment sur les obligations d'application des accords de paix (de Dayton et d'Ohrid), et sur le devoir de coopération avec le Tribunal pénal international pour l'exYougoslavie (TPIY) ${ }^{12}$.

12 Globalement, le PSA ne constitue donc pas uniquement un cadre pour préparer les pays des Balkans occidentaux à l'adhésion à l'UE par une convergence institutionnelle et légale, mais forme, en parallèle, un instrument pour la construction et la consolidation des États. Des dispositions visant à la sécurité et à la stabilité des Balkans occidentaux sont ainsi contenues dans le volet politique du PSA lui-même, dépassant le seul cadre de la PESC.

\section{De la stabilisation vers l'association}

13 Malgré des problèmes de stabilité persistants, l'inflexion du PSA se déplace pourtant progressivement vers la dimension de l'association. Ainsi, l'agenda issu du sommet de Thessalonique de juin 2003 vise à la transition de l'approche de stabilisation vers une approche plus centrée sur la préparation à l'adhésion à l'UE :

$\mathrm{Au}$ cours des quatre dernières années, la politique de stabilisation et d'association de l'Union européenne a contribué de manière cruciale à promouvoir la stabilité à travers la région et à la rapprocher d'elle. Cette politique doit désormais être renforcée et enrichie d'éléments du processus d'élargissement afin de pouvoir mieux faire face aux défis qui résultent de la transition qu'opèrent ces pays depuis l'étape de la stabilisation et la reconstruction, vers leur développement durable ainsi que l'association avec l'UE et leur intégration en elle ${ }^{13}$.

14 Tandis que la déclaration confirme la perspective européenne des Balkans occidentaux et met au premier plan le développement durable, force est de constater que la stabilisation politique de la région occupe jusqu'à ce jour une place importante dans l'agenda européen.

15 Si la situation au Kosovo, où la communauté internationale poursuit sa présence à la suite de la déclaration d'indépendance de février 2008, est généralement stable, les incidents violents entre populations serbe et albanaise dans le nord de Mitrovica au tournant 2008/2009 ont démontré la fragilité de la situation sécuritaire. Par ailleurs, la cohabitation des trois peuples constituants de la Bosnie-Herzégovine souffre d'un repli identitaire accentué. Les autorités politiques bosniaques, croates et serbes peinent, malgré des efforts internationaux renouvelés, à se mettre d'accord sur une réforme 
constitutionnelle qui leur permettrait de surmonter l'ordre daytonien et les fréquents blocages. Par conséquent, la fermeture du Bureau du Haut Représentant (OHR), quoique débattue au cours des dernières années avec une impatience croissante de la part de l'UE, se heurte toujours à l'absence d'une situation politique stable.

En Macédoine, la coexistence des communautés macédonienne et albanaise demeure fragile. Au demeurant, les conflits bilatéraux persistent, notamment par rapport à la délimitation des frontières et au retour des réfugiés. Dès lors, malgré une certaine transition de la stabilisation vers l'association, la tension originelle entre endiguement et transformation demeure d'actualité.

\section{La politique de conditionnalité entre stabilité et crédibilité}

17 La double exigence à la fois d'une stabilisation et d'une association plus étroite des Balkans occidentaux présentée plus haut pose un défi majeur à l'action de l'Union européenne dans la région. Si l'on peut considérer, d'une part, qu'une situation politique et sécuritaire stable est une condition préalable indispensable au succès de l'intégration européenne et, d'autre part, que l'intégration européenne est créatrice de stabilité à long terme, on peut néanmoins s'interroger sur la complémentarité de ces deux approches. En effet, le double objectif de l'UE dans les Balkans occidentaux risque de compliquer, voire d'entraver la mise en œuvre d'une politique d'élargissement cohérente.

En principe, la conditionnalité offre un cadre clairement défini à l'association indiquant les conditions à remplir avant d'entrer dans la prochaine phase de rapprochement à l'UE. Étape fondamentale dans l'association avec l'Union européenne, la signature d'un ASA est soumise au respect de conditions générales, auxquelles s'ajoutent éventuellement d'autres conditions spécifiques à chaque pays. Comme le remarque la Commission dans sa stratégie d'élargissement en 2005, l'association à travers un ASA requiert aussi un certain degré de stabilisation préalable ${ }^{14}$. Par ailleurs, une fois le statut de candidat obtenu, des négociations d'adhésion ne peuvent être ouvertes qu'en cas de respect des critères politiques de Copenhague ${ }^{15}$. Les conditions et critères de référence définis par l'UE confèrent dès lors au processus une grande transparence et prévisibilité.

19 Une analyse de la mise en œuvre de la politique d'élargissement révèle pourtant que le respect de la conditionnalité n'est pas le seul paramètre pris en compte dans les décisions sur la progression des pays de la région vers l'association. Le danger de déstabilisation politique persistant au cours des dernières années rend ainsi tout aussi nécessaire la prise en compte de la dimension stabilisatrice. Certes, le refus du Conseil de reconnaître le statut de candidat à un pays, la décision de ne pas ouvrir ou de suspendre des négociations face au non-respect des critères d'adhésion ou le refus de certains États membres de ratifier un accord peuvent être justifiés au nom du respect de la conditionnalité. Une mise en œuvre démesurément stricte de celle-ci risque cependant d'avoir des effets négatifs sur la situation politique d'un pays aspirant à l'adhésion. Comme la dynamique de réformes dépend en large partie de l'existence d'une perspective crédible d'entrer dans l'UE, seul le maintien de cette crédibilité permet à des gouvernements souvent fragiles de s'imposer face à des oppositions sceptiques et, dans certains cas, plus intéressées par des considérations ethniques ou 
nationalistes. Bien que le consensus sur la perspective d'adhésion unisse la grande majorité des acteurs politiques dans la région, le coût des réformes exigées demeure relativement élevé, et peut dès lors polariser le paysage politique. Une incitation constante et des progrès visibles paraissent ainsi indispensables pour maintenir l'élan de la classe politique et pour renforcer l'approbation des populations, qui doivent accepter des restructurations parfois douloureuses, notamment du secteur économique, sans pour autant recevoir de contrepartie positive immédiate de leurs efforts. Il est de la sorte important de soutenir l'approche méritocratique, où chaque pays est compensé pour ses efforts individuels, tout en réfléchissant à de possibles compensations qui permettront de maintenir le soutien à l'objectif de l'adhésion à un niveau élevé, aussi bien pour les élites politiques que pour la population en général ${ }^{16}$. Tandis qu'une application trop stricte de la conditionnalité risque alors d'affaiblir le consensus européen, on peut craindre de l'autre côté que l'assouplissement trop fréquent de la conditionnalité dû à des considérations de stabilisation ne diminue la crédibilité de cet outil et, par conséquent, réduise l'effet de levier de l'Union sur les pays de la région.

En somme, on peut donc constater un double risque de décrédibilisation : d'une part, la décrédibilisation de la conditionnalité si le maniement trop souple des critères d'adhésion crée l'impression que l'association avec l'UE est possible en l'absence de réels progrès dans les réformes; d'autre part, la décrédibilisation de la perspective d'adhésion si l'interprétation rigoureuse de la conditionnalité crée la perception que l'on n'avance plus dans le processus d'association ou que le prix à payer pour l'adhésion est trop élevé par rapport aux gains. Les deux mécanismes risquent fort de freiner la volonté d'un pays à poursuivre des réformes. Par conséquent, les effets potentiellement déstabilisateurs d'une conditionnalité strictement respectée ou d'une conditionnalité assouplie qui réduirait comme corollaire l'incitation aux réformes, font en sorte que l'UE oscille de façon permanente entre politiques de stabilité et de transformation. La mise en œuvre de la politique de conditionnalité apparaît dès lors souvent peu uniforme ${ }^{17}$.

21 Des études de cas du déroulement du PSA dans trois pays engagés dans l'élargissement européen, la Macédoine, la Bosnie-Herzégovine et la Serbie, permettra de dégager les principales dynamiques à l'œuvre dans la formulation de la politique européenne envers les Balkans, et facilitera l'évaluation de l'efficacité de la double approche de l'UE.

\section{Macédoine}

Avec la signature d'un accord de stabilisation et d'association le 9 avril 2001, la Macédoine est le premier pays des Balkans occidentaux à établir une telle relation contractuelle avec l'UE. Si l'on considère pourtant les circonstances accompagnant la conclusion de l'accord, on constate que, loin de marquer « le passage de la stabilisation à l'association $»^{18}$, celle-ci vise, avant tout, à rétablir la stabilité dans un climat d'affrontements interethniques entre la population macédonienne slave et la minorité albanophone du pays. En plein milieu d'une crise politique et de sécurité grave, l'UE conclut l'ASA avec la Macédoine dans le souci d'éviter la dégradation du conflit et de prévenir ainsi un risque de déstabilisation de toute la région. 

de stabilisation entrepris par la communauté internationale sous l'égide des États-Unis et de l'UE. Après sa paralysie face aux guerres yougoslaves des années 1990, cette dernière doit faire preuve de sa meilleure capacité de gestion des crises. Au cœur des efforts de stabilisation se trouve la médiation de l'UE et des États-Unis dans la conclusion de l'Accord-cadre d'Ohrid en août 2001, qui met fin aux hostilités et définit un large programme de mesures incluant des réformes constitutionnelles, la décentralisation administrative et la réorganisation de la police. La supervision de ces accords incombe au Représentant spécial de l'UE déployé en juin 2001. Par ailleurs, la mission de l'OTAN mise en place en octobre 2001 est remplacée à partir d'avril 2003 par la mission militaire Concordia, première mission de ce type déployée par l'UE dans le cadre de la PESC. En décembre 2003, celle-ci est suivie par la mission de police Proxima qui compte un effectif de 200 policiers des États membres. si la signature de l'ASA peut alors être considérée comme un succès quant à son effet stabilisateur, la conclusion de l'accord à ce moment précis ne résulte toutefois pas du progrès dans le programme de réformes. L'on peut ainsi affirmer que «l'accord avec la Macédoine a été conclu, non parce que ce pays remplissait les critères politiques et économiques prévus, mais parce que les Européens souhaitaient consolider le seul pays de la région où la cohabitation entre les différentes communautés semblait ne pas soulever trop de difficultés $»^{19}$.

La prééminence du volet de stabilisation se révèle aussi dans l'orientation de l'ASA. Tandis que l'accord conclu avec la Croatie en octobre 2001 se caractérise par une logique de pré-adhésion, dans le cas de la Macédoine, c'est l'objectif de stabilisation qui est privilégié. Par ailleurs, la période de transition prévue dans les ASA pour la mise en œuvre de l'association avec l'UE s'élève pour la Croatie à six ans, et pour la Macédoine à une phase plus longue de dix ans. Les deux accords reflètent dès lors un degré d'avancement sur la voie de l'intégration européenne bien différent ${ }^{20}$.

Malgré cette période de transition assez longue et avant même l'entrée en vigueur de son ASA le $1^{\mathrm{er}}$ avril 2004, la Macédoine dépose sa candidature à l'adhésion à l'UE le 22 mars de la même année. Quoique l'initiative macédonienne soit estimée prématurée par bon nombre de commentateurs tant à Bruxelles qu'à Skopje, la Commission recommande en novembre 2005 l'octroi par le Conseil du statut de candidat à la Macédoine. Le bilan dressé par la Commission sur les avancées dans le processus de stabilisation et d'association reste toutefois mitigé. Ainsi, l'avis atteste que le pays est "en bonne voie pour respecter les critères politiques $\|^{21}$. Quant aux critères économiques de Copenhague, la Commission constate que le pays n'est pas capable de résister à moyen terme, c'est-à-dire dans une période de cinq ans, aux pressions concurrentielles et aux forces du marché intérieur de l'Union. Néanmoins, l'avis souligne qu'il le sera dans le futur à condition que les réformes économiques engagées soient poursuivies. Quant à l'adoption de l'acquis communautaire, l'avis estime que des efforts considérables sont requis pour que «la plupart des obligations liées à l'adhésion » soient assumées à moyen terme ${ }^{22}$.

27 Outre l'ambiguïté de l'avis par rapport à l'évaluation des progrès accomplis, des réflexions sur la stabilisation de la Macédoine quatre ans après les affrontements interethniques survenus en 2001 occupent une place importante dans le document. Ainsi, la Commission constate que les progrès accomplis dans l'application de l'accord- 
cadre d'Ohrid "se sont avérés essentiels pour le renforcement de la stabilité du pays » et que la mise en œuvre effective du nouveau cadre législatif « consolidera davantage le climat de confiance et de stabilité du pays et engendrera un environnement de plus en plus favorable à l'intégration à l'UE $»^{23}$.

Si le Conseil européen du 16 décembre 2005 décide finalement de conférer à la Macédoine le statut de candidat, il paraît alors que le facteur décisifconduisant à cette réponse positive à la demande macédonienne ne soit pas le progrès dans les réformes politiques et économiques, mais bien la fragilité persistante de la situation politique et interethnique. En effet, l'on craint que l'instabilité du pays ne soit exacerbée par un refus du Conseil, d'autant plus que les négociations sur le statut du Kosovo débutant en novembre 2005 comportent le risque d'une déstabilisation régionale. Comme en 2001 pour la conclusion de l'ASA, des considérations de stabilisation pèsent alors de manière tout aussi lourde sur la décision de l'UE dans cette deuxième phase d'association.

Bien que la Macédoine ait obtenu le statut de pays candidat en 2005, et contrairement aux deux autres candidats à l'adhésion - Croatie et Turquie -, les négociations d'adhésion n'ont jusqu'à ce jour pas été ouvertes avec Skopje. Le respect des critères politiques de Copenhague étant un préalable essentiel au lancement des négociations, l'UE a renoncé à définir une date avant qu'un «niveau suffisant de conformité aux critères d'adhésion » ne soit atteint ${ }^{24}$. Ainsi, le nouveau Partenariat européen de février 2008 fixe huit critères de référence (« opening benchmarks») à remplir afin qu'une date pour l'ouverture des négociations puisse être fixée. Ces critères concernent des domaines divers tels que le dialogue politique, la mise en œuvre effective de la loi sur la police, la lutte contre la corruption, les réformes judiciaires et administratives, la lutte contre le chômage ainsi que l'amélioration du climat général des affaires ${ }^{25}$. Par ailleurs, la coexistence pacifique et la coopération entre les deux communautés demeurent fragiles, comme le montrent lestensions précédant les élections anticipées de juin 2008. À ces tensions interethniques viennent se rajouter des incidents entre les deux principaux partis albanais, l'Union démocratique pour l'intégration et le Parti démocratique des Albanais, révélateurs de rivalités et de conflits intra-ethniques.

Saluant les avancées en matière de réformes, la Commission recommande finalement en octobre 2009 l'ouverture des négociations d'adhésion ${ }^{26}$. Face à l'opposition ferme de la Grèce du fait de son désaccord avec la Macédoine sur l'appellation officielle du pays, les ministres des Affaires étrangères de l'UE décident toutefois en décembre 2009 de reporter à l'année 2010 la détermination d'une date pour le lancement des négociations.

31 En somme, le cas macédonien est donc révélateur de la prévalence de l'objectif de stabilisation du PSA en présence d'une situation précaire de construction nationale. Néanmoins, même si l'UE a assoupli à certains moments son exigence d'un respect rigoureux de la conditionnalité, le fait que la Macédoine en tant que pays candidat attende depuis quatre ans l'ouverture des négociations d'adhésion montre que cette indulgence temporaire ne dispense pas de l'obligation à mener les réformes nécessaires pour atteindre les standards politiques et économiques liés à l'adhésion. La double approche de l'UE semble ainsi alterner en fonction de la situation sur place, tout en maintenant un certain équilibre entre ses deux objectifs de stabilisation et d'association. 


\section{Bosnie-Herzégovine}

32 Tandis que les tensions interethniques posent un défi à l'association européenne de la Macédoine, elles sont source d'un blocage quasiment perpétuel en Bosnie-Herzégovine. Les structures politiques en Bosnie-Herzégovine, qui reposent sur la constitution élaborée dans le cadre des accords de Dayton, reflètent largement le résultat d'une guerre caractérisée par le nettoyage ethnique et la volonté de conquérir le plus de territoire possible pour son propre groupe ethnique. Alors que la violence physique appartient heureusement au passé, les clivages partisans persistent dans la vie politique et des tensions interethniques prennent souvent le dessus sur une vision stratégique à plus long terme. Lors des élections de 2006 et 2008, l'appel politique à l'ethno-nationalisme s'est encore renforcé. Bien que les trois principaux groupes ethniques sur place - Croates, Musulmans et Serbes - partagent selon les sondages l'objectif commun de l'adhésion de leur pays à l'Union européenne ${ }^{27}$, le quotidien politique est dominé par des disputes largement futiles, faisant en sorte que le progrès nécessaire en matière de réformes constitutionnelles, préalable pourtant indispensable à l'octroi du statut de candidat, semble compromis.

Globalement, le problème fondamental de la Bosnie-Herzégovine consiste en l'absence de sa pleine souveraineté extérieure, due à la forte présence internationale nécessaire au maintien de la stabilité. En effet, le Bureau du Haut Représentant de la communauté internationale (OHR), malgré de nombreuses annonces contraires, n'a toujours pas pu terminer son mandat. Le Conseil pour la réalisation de la Paix (PIC: Peace Implementation Council), composé des principaux acteurs extérieurs impliqués dans la stabilisation du pays, vient de repousser au mois de février 2010 la possible décision de son départ ${ }^{28}$. Par ailleurs, la mission EUFOR Althea, bien que réduite en effectifs, vient de voir son mandat prolongé afin de continuer à veiller sur la sécurité dans le pays et à prévenir tout nouvel éclatement de violence. La situation de la Bosnie-Herzégovine est donc très particulière, avec des effets correspondants sur les aspirations européennes du pays.

Les négociations en vue d'un accord de stabilisation et d'association ne débutent qu'en janvier 2006 dans un climat tendu, qui ne sera que renforcé par le grand interventionnisme du nouveau Haut Représentant, Christian Schwarz-Schilling. En février 2007, à l'occasion de la prise de pouvoir d'un nouveau gouvernement en BosnieHerzégovine, le Commissaire Olli Rehn signale que la Commission n'envisage pas de conclure l'ASA en l'absence d'une réforme de la police et d'une meilleure coopération avec le TPIY, et qu'il s'attend à ce que le nouvel exécutif fasse des " progrès concrets » sur les questions encore ouvertes ${ }^{29}$. Le 4 décembre 2007, un accord de principe sur la réforme de la police est atteint, et l'ASA paraphé en conséquence. Toutefois, la signature finale de l'accord est conditionnée par la mise en œuvre effective du plan de réformes esquissé. Ce plan n'est finalement approuvé par le Parlement bosniaque qu'en avril 2008. La signature de l'ASA a lieu le 16 juin 2008, ce qui fait de la BosnieHerzégovine le dernier pays de la région à établir des relations contractuelles avec l'Union.

En raison de la faible capacité administrative de la Bosnie-Herzégovine et du fait de sa souveraineté limitée, l'UE doit souvent compter sur la coercition pour imposer les réformes nécessaires. En l'absence de structures politiques fonctionnelles au niveau fédéral, la conditionnalité classique demeure, même dans sa forme stricte, trop faible 
pour se prévaloir face aux blocages intérieurs. C'est ainsi que l'UE a dû accepter une version simplifiée de la réforme de la police, qui correspond à peine aux exigences minimales formulées par l'Union ${ }^{30}$. L'idée de stabilisation prévaut ainsi encore clairement en Bosnie-Herzégovine, où l'association est largement bloquée par les désaccords entre les dirigeants des deux entités, la Republika Srpska d'un côté, et la Fédération bosno-croate de l'autre. La transition de l'objectif de stabilisation vers une véritable association passe par une réforme constitutionnelle, qui constitue l'une des conditions définies par le PIC avant la fermeture de l'OHR. Or, la première tentative de doter le pays d'une structure institutionnelle plus efficace a échoué de peu en 2006. Actuellement, le processus de Butmir sous l'égide des États-Unis et l'Union européenne vise à unir les différents partis bosniaques autour d'un compromis qui permettrait la création de structures gouvernementales viables, ce qui faciliterait le départ du Haut Représentant et le passage de la pleine responsabilité du pays entre les mains des Bosniens. Cependant, l'issue de ces pourparlers n'est pas claire, et il semble loin d'être acquis que les différents acteurs réussiront cette fois-ci à se mettre d'accord sur l'avenir du pays.

En définitive, la dimension de stabilisation est encore nettement dominante en BosnieHerzégovine. En raison de la faiblesse des structures gouvernementales centralisées, la conditionnalité classique peine à s'imposer, et l'action de l'OHR demeure cruciale pour l'avancement des réformes.

\section{Serbie}

Le difficile arbitrage entre l'application stricte d'une conditionnalité préétablie et un assouplissement des exigences motivé par des considérations politiques devient particulièrement apparent dans le cas de la Serbie. En effet, l'on constate dans le temps latransformation d'une attitude particulièrement exigeante de l'Union européenne envers cet État-clé de la région, et dont la responsabilité dans les violences des années 1990 est incontestablement l'une des plus lourdes, vers une plus grande souplesse à la suite de la déclaration unilatérale d'indépendance du Kosovo en février 2008.

Aussi est-ce envers la Serbie que la Commission a fait usage de toute la latitude politique à sa disposition dans l'application du PSA : après un lancement tardif des négociations en vue de la conclusion d'un ASA en 2005, à ce moment-là encore avec la Fédération de Serbie-Monténégro, le manque de coopération avec le TPIY entraîne la suspension de ces pourparlers à partir du 3 mai 2006 et jusqu'en juin 2007. Cette mesure drastique, prise à quelques semaines seulement du référendum sur l'indépendance monténégrine, peut surprendre après les efforts considérables qu'avait déployés l'UE pour conserver la fédération entre les deux dernières républiques de l'ancienne Yougoslavie qui demeuraient unies. Ainsi, c'était sous la pression du Haut Représentant pour la PESC Javier Solana qu'avait été négocié en mars 2002 l'accord de Belgrade prévoyant la création de l'Union de Serbie-Monténégro ${ }^{31}$. Or, la suspension des négociations en vue d'un ASA ne pouvait que gonfler les rangs des indépendantistes auMonténégro et précipiter l'éclatement de la dernière incarnation d'une unité yougoslave $^{32}$; au lieu d'assouplir ses conditions en vue du référendum monténégrin, l'UE les maintient, tout en fixant le pourcentage de suffrages nécessaires pour atteindre l'indépendance du Monténégro à $55 \%$ dans l'espoir de prévenir ainsi la sécession. Le « oui » remportant le suffrage avec 55,5 \% des voix, l'UE doit cependant reconnaître la 
création d'un nouvel État dans la région et poursuit désormais des négociations bilatérales avec les deux entités de manière séparée.

Toutefois, la Commission se rend rapidement compte des risques d'une marginalisation de la Serbie au sein du processus de stabilisation et d'association. Dans sa stratégie d'élargissement 2006-2007, publiée en novembre 2006, elle souligne que «la Commission ne sous-estime pas l'ampleur des enjeux auxquels elle [la Serbie] est confrontée dans son contexte politique actuel $»^{33}$ et prévoit une reprise des négociations dès que la Serbie aura rempli la condition de pleine coopération avec le TPIY. Les pourparlers pour un ASA avec Belgrade reprennent ainsi en juin 2007 suite à l'élection d'un nouveau gouvernement pro-européen. Bien que la pleine coopération avec le Tribunal pénal international ne soit pas à ce moment-là estimée atteinte, l'arrestation du fugitifZdravko Tolimir en mai 2007 sur le territoire de BosnieHerzégovine est considérée comme un signe suffisant de coopération de la part de Belgrade. La Commission décide ainsi d'attendre qu'une certaine stabilisation vienne du pays lui-même avant de s'engager plus loin dans l'association.

Ce sont les négociations sur le statut du Kosovo, menées sous l'égide de la troïka ÉtatsUnis-Russie-UE, qui introduisent une nouvelle orientation dans l'approche de l'Union envers la Serbie. En effet, l'engagement hâtif du Président américain George W. Bush en faveur d'une déclaration unilatérale d'indépendance en cas d'échec des pourparlers multilatéraux rend probable dès l'été 2007 une solution largement défavorable à Belgrade. Dans le souci de prévenir une déstabilisation intérieure de la Serbie, la Commission précipite les pourparlers et paraphe l'ASA avec la Serbie en novembre 2007, en vue d'une signature en février 2008. Dans une conférence de presse du 6 février 2008, moins de deux semaines avant la date pressentie de la déclaration d'indépendance du Kosovo, le Commissaire pour l'élargissement alors en place, Olli Rehn, souligne que «l'UE est engagée à accélérer la route de la Serbie vers l'Europe ».34 Ainsi, l'UE propose dès fin janvier 2008 un accord politique intérimaire, qui contiendrait beaucoup des clauses prévues dans l'ASA. Toutefois, cette proposition est rejetée par le Premier ministre d'alors, Vojislav Koštunica, qui y voit une récompense pour accepter la perte imminente du Kosovo, lequel déclarera finalement son indépendance unilatéralement le 17 février 2008. S'enfonçant de plus en plus dans une attitude nationaliste, Koštunica refuse par le même argument de signer l'ASA pourtant paraphé, et préfère provoquer des élections législatives anticipées, en quittant avec son parti la coalition gouvernementale.

Dans les semaines qui précèdent les élections du 11 mai 2008, l'UE redouble d'efforts pour stabiliser une Serbie dont le paysage politique est sérieusement ébranlé par la sécession de sa province méridionale. Le Parti démocratique de Serbie de Koštunica fait des avances au Parti radical nationaliste, dont le candidat à la Présidence a obtenu plus de voix que le leader démocratique Boris Tadić. De son côté, l'UE craint un retour à l'isolation de la Serbie dans le cas d'un gouvernement eurosceptique. Ainsi, la signature de l'ASA - par le Président Tadić et le Vice-Premier ministre Đelić - a lieu le 29 avril 2008 au beau milieu de la campagne électorale, malgré une coopération toujours décevante avec le TPIY. Les États membres accordent début mai des visas gratuits à certains groupes de la population serbe - dont les étudiants -, souhaitant renforcer le camp pro-démocratique et pro-européen.

Les efforts de l'Union paient, et les élections donnent une majorité claire à la coalition «Pour une Serbie européenne ». Quelques semaines seulement après la formation du 
gouvernement, le fugitif Radovan Karadžić est appréhendé et transféré au TPIY de La Haye, dans une opération qui semble confirmer l'importance d'un acte symbolique de soutien de la part de l'UE. La signature de l'ASA, étape fondamentale du PSA, symbolise donc dans le cas de la Serbie la transition d'une approche stricte vers une approche plus souple de la part de l'Union. Craignant une déstabilisation suivie d'un revirement politique en Serbie après l'indépendance du Kosovo, l'UE a voulu affirmer la vocation européenne du pays en espérant compenser en quelque sorte le sentiment de Belgrade que son intégrité territoriale a été violée. L'arrestation de Karadžić semble confirmer le bien-fondé de cet assouplissement de la conditionnalité, tout comme la demande du statut de candidat officiel que vient de déposer legouvernement serbe à Bruxelles fin décembre 2009.

En somme, le cas de la Serbie illustre le succès d'une application pragmatique du principe de conditionnalité : en assouplissant ses exigences à la suite de la sécession du Kosovo, laCommission a empêché une déstabilisation de la Serbie et a en échange renforcé le camp démocratique et modéré, qui s'est depuis engagé de manière claire sur la voie des réformes.

\section{Conclusion}

L'objectif ambitieux du processus de stabilisation et d'association est double: d'une part, il vise à la stabilisation post-conflit d'une région déchirée par la violence il y a à peine quinze ans; d'autre part, il tente une transformation par une association plus étroite avec l'UE en vue d'une adhésion à terme qui viendrait cimenter les progrès accomplis. La conditionnalité, grande réussite lors de l'élargissement à l'Europe centrale et orientale, forme l'outil central de cette tentative. Or, une analyse plus précise des chemins parcourus par les pays des Balkans occidentaux révèle un bilan mitigé du PSA jusqu'à présent. Aussi semblerait-il que la stabilité est un préalable pour le fonctionnement du principe méritocratique contenu dans la conception du PSA.

Ainsi, s'il existe des contraintes par rapport à la stabilisation du pays à prendre en compte, un assouplissement de la conditionnalité peut paraître indispensable. Comme nous venons de le voir, l'UE s'est vue à plusieurs reprises obligée de naviguer entre deux approches parfois contradictoires ou pour le moins difficilement conciliables : d'un côté, celle d'une stabilisation et d'un renforcement de la sécurité, et de l'autre, l'impératif d'impulser le développement d'une région qui demeure généralement encore loin, au niveau politique aussi bien qu'économique, des standards européens exigés à tout pays candidat à l'adhésion.

En somme, la nécessité de prendre en compte l'agenda de stabilisation amène donc souvent l'UE à conduire les politiques d'intégration dans une logique de stabilisation plutôt que dans une logique de récompense pour les progrès accomplis dans la mise en œuvre des réformes politiques, institutionnelles et économiques. En conséquence, le besoin de stabilisation immédiate tend à prendre le pas sur la stabilité durable, qui peut être atteinte uniquement par une transformation réelle de la situation sur place.

Toutefois, même si l'on peut critiquer une application peu uniforme de la conditionnalité, ce qui peut paraître comme incohérence stratégique résulte finalement de la nécessité de trouver un équilibre entre les considérations de stabilisation et de transformation. En effet, il existe des exemples d'un assouplissement des exigences de l'Union uniquement dans le but de désamorcer un potentiel conflictuel. Néanmoins, il 
ne faut pas méconnaître la réelle capacité de stabilisation de certaines concessions accordées au sein du PSA. Sans doute, les efforts de l'UE pour nouer des relations plus étroites avec la Macédoine au moment des dérives intercommunautaires à travers la signature de l'ASA en 2001 et son accompagnement dans l'élaboration et la mise en œuvre de l'accord-cadre d'Ohrid contribuaient-ils essentiellement à l'amélioration de la situation politique et sécuritaire $d u$ pays. À l'heure actuelle, l'équilibre intercommunautaire demeure fragile mais relativement stable, et ceci malgré le potentiel de déstabilisation qu'aurait pu avoir l'indépendance du Kosovo voisin. Le fait que la Macédoine attende depuis l'obtention du statut de candidat en 2005 l'ouverture des négociations d'adhésion révèle cependant que l'assouplissement de la conditionnalité à l'égard d'un pays candidat ou d'un candidat potentiel n'est que temporaire ou partiel. Si un pays dans certaines circonstances ne doit pas pleinement et immédiatement remplir les critères définis par l'UE pour atteindre la prochaine étape du PSA, il ne pourra cependant pas durablement renoncer à mettre en œuvre le programme des réformes nécessaires à son intégration européenne.

Enfin, il est important de relever que ce ne sont pas uniquement les tentatives de stabilisation ou le progrès des réformes qui déterminent l'avancement d'un pays sur le chemin de l'adhésion. Trois autres facteurs cruciaux peuvent avoir un impact considérable sur l'effet de levier exercé par la politique de conditionnalité. Premièrement, à l'intérieur d'un pays, la volonté des dirigeants politiques à mettre en œuvre l'agenda des réformes, voire leur capacité à le faire, jouent un rôle primordial.La situation en Bosnie-Herzégovine illustre ce constat: malgré une conditionnalité assouplie et des réformes plus ou moins imposées par l'Union européenne et par le Haut Représentant ${ }^{35}$, les luttes intestines entre les différents acteurs politiques minent des progrès réels et ont récemment empêché la libéralisation du régime des visas, pourtant accordée à la Serbie, au Monténégro et à la Macédoine. Deuxièmement, le comportement de certains États membres de l'Union peut avoir un impact non négligeable sur l'avancement des négociations. Ainsi, l'on a constaté plusieurs exemples de blocages du processus d'association motivés par des litiges bilatéraux opposant un État membre à un pays candidat ou potentiellement candidat. Le véto slovène à l'encontre de la Croatie à cause d'une dispute sur la délimitation des frontières entre les deux pays, le véto néerlandais à l'encontre de la Serbie ainsi que l'opposition grecque à l'adhésion de la Macédoine à l'OTAN ou l'UE en raison du nom constitutionnel contesté de Skopje ne sont que quelques exemples d'un État membre profitant de l'asymétrie des négociations pour résoudre un conflit bilatéral. Finalement, sans lien direct avec les pays des Balkans occidentaux, les incertitudes récentes sur l'entrée en vigueur du traité de Lisbonne ainsi que les débats concernant la capacité d'absorption de l'UE sont déterminants pour la vitesse de l'intégration des États de la région au sein de l'Union.

1.

2. 


\section{NOTES}

Notamment par le déploiement de missions militaires (EUFOR Althea en Bosnie-Herzégovine depuis décembre 2004, Concordia en Macédoine de mars à décembre 2003) et des missions de police (EUPM en Bosnie-Herzégovine depuis janvier 2003, Proxima en Macédoine de décembre 2003 à décembre 2005) ou civiles (notamment EULEX, opérationnel depuis décembre 2008 au Kosovo.

Voir Ragaru (Nadège), «Le défi d'un nouvel élargissement de l'UE », Questions internationales,23 (1-2), 2007, p. 34.

3. En raison du débat autour du nom de la Macédoine contestée par la Grèce depuis la déclaration d'indépendance de la République yougoslave en novembre 1991, le nouvel État a intégré les Nations Unies en 1993 sous le nom d'Ancienne République yougoslave de Macédoine (ARYM). Tandis que les Nations Unies, l'UE et l'OTAN entretiennent des relations avec le pays sous ce nom, 127 pays dans le monde ont jusqu'à ce jour reconnu l'Etat macédonien sous le nom constitutionnel « République de Macédoine », parmi lesquels les États-Unis, la Grande-Bretagne, la Russie, la Chine ainsi que toutes les anciennes républiques yougoslaves. Nous utiliserons par la suite le nom Macédoine, moins encombrant, sans que cela implique une prise de position.

4. Conseil européen. «Conclusions de la présidence », DOC-99-2, Cologne, 03-04/06/99, § 72.

5. Sommet UE - Balkans occidentaux, « Déclaration », 10229/03, Thessalonique, 21/06/03, § 2.

6. Le statut du Kosovo demeure ambigu au sein de l'UE: le Conseil a clairement affirmé la perspective européenne de l'ancienne province serbe; toutefois, comme seulement 22 États membres sur 27 ont jusqu'à ce jour reconnu la déclaration unilatérale d'indépendance de Pristina, le Conseil a élaboré un partenariat européen pour le Kosovo, mais celui-ci demeure pour l'instant en-dehors des structures du PSA. L'UE est par ailleurs présente à travers sa mission de justice EULEX ainsi que par le Représentant spécial envoyé à Pristina.

7. Pop (Valentina), «EU lifts hurdle on Serbia's path to accession », EU Observer, 08/12/09.

8. « Tadić to submit EU candidate status bid », B92, 21/12/09, à l'adresse : [http://www.b92.net/ eng/news/politics-article.php?yyy=2009\&mm=12\&dd=21\&nav_id=63930]

9. Rehn (Olli), «Lessons from EU enlargement for its future foreign policy»,discours au European Policy Centre, SPEECH/09/492, Bruxelles, 22/10/09. Toutefois, la Slovénie a récemment bloqué l'ouverture de trois nouveaux chapitres. Voir Pop (Valentina), « Slovenia still holding back Croatia on EU path ", EU Observer, 22/12/09.

10. Traité sur l'Union européenne, art. 49.

11. Voir par exemple Altmann (Franz-Lothar), «EU und Westlicher Balkan : Eine schwierige Verlobung » [L'UE et les Balkans occidentaux : des fiançailles difficiles], Südosteuropa, 53 (2), 2005, p. 205 ; Drouet (Michel), « La perspective de l'intégration des Balkans occidentaux á l'UE comme modalité de leur stabilité politique », in Drouet (Michel), Richet (Xavier), éds., Vers l'élargissement de l'Union européenne à l'Europe du Sud-Est, Rennes : Presses universitaires de Rennes, 2007, p. 157 ; Terrenoire (Alain). «Les Balkans occidentaux et l'Union européenne» Journal officiel de la République française. Avis et rapports du Conseil économique et social, (23), 29/11/07, p. 56 ; Grabbe (Heather), The EU's Transformative Power: Europeanization through Conditionality in Central and Eastern Europe, New York : Palgrave Macmillan, 2006.

12. Voir Pridham (Geoffrey), « Democratizing the Western Balkans: Challenges and Burdens for the European Union", International Issues/Slovak Foreign Policy Analysis, 17 (3), 2008 ; Terrenoire (Alain), art.cit., p. 29.

13. Conseil de l'UE, «L'agenda de Thessalonique pour les Balkans occidentaux : Progresser sur la voie de l'intégration européenne ", $2518^{\mathrm{e}}$ session du Conseil, Relations extérieures, 10369/03, Luxembourg, 16/06/03, annexe A. [texte francisé par la rédaction de Balkanologie]. 
14. Commission européenne, «Document de stratégie pour l'élargissement 2005 », COM (2005) 561 final, Bruxelles, 09/11/05.

15. Rapoport (Cécile), « Les accords de stabilisation et d'association, instruments de stabilisation et de pré-adhésion », in Drouet (Michel), Richet (Xavier), éds., op. cit., pp. 178ss.

16. Drouet (Michel), art.cit., pp. 161-163.

17. Voir aussi Elbasani (Arolda), «The Stabilisation and Association Process in the Balkans: Overloaded Agenda and Weak Incentives? », EUI Working Paper SPS, (3), 2008, p. 13.

18. Rapoport (Cécile), art.cit., p. 179.

19. Heimerl (Daniela), «UE-Balkans occidentaux. Après le sommet de Thessalonique de juin 2003 ", Le Courrier des Pays de l'Est, (1039), septembre 2003, p. 34.

20. Rapoport (Cécile), art.cit., p. 177.

21. Commission européenne, "Avis de la Commission concernant la demande d'adhésion à l'Union européenne présentée par l'ancienne République yougoslave de Macédoine », COM (2005) 562 final, Bruxelles, 09/11/05.

22. Ibid.

23. Ibid.

24. Ibid.

25. Conseil de l'UE, « Décision du Conseil 2008/212/CE du 18 février 2008 relative aux principes, aux priorités et aux conditions figurant dans le partenariat pour l'adhésion avec l'ancienne République yougoslave de Macédoine et abrogeant la décision 2006/57/CE », OJ 2008 L 80/32.

26. Commission européenne, "Stratégie d'élargissement et principaux défis 2009-2010", $\operatorname{COM}(2009)$ 533, Bruxelles, 14/10/09.

27. Gallup Balkan Monitor 2008, http://www.balkan-monitor.eu/.

28. Bureau du Haut Représentant, communiqué, Sarajevo, 29 novembre 2009, à l'adresse : [http://www.ohr.int/print/?content_id=44144]

29. Rehn (olli), communiqué, 9 février 2007, à l'adresse: [http://ec.europa.eu/ commission_barroso/rehn/press_corner/statements/statements_2007_en.htm]

30. Vogel (Toby), « Bosnia removes last hurdle to pact with EU », European Voice, 11/04/08, à l'adresse : [http://www.europeanvoice.com/current//article/2008/04/713april/bosnia-removeslast-hurdle-to-pact-with-eu/60279.aspx]

31. Lukić (Reneo), "From the Federal Republic of Yugoslavia to the Union of Serbia and Montenegro », in Ramet (Sabrina P.), Pavlaković (Vjeran), eds., Serbia since 1989 : Politics and Society under Milošević and After, Washington : University of Washington Press, 2005, p. 81.

32. Drouet (Michel), art.cit., pp. 165s. ; Bieber (Florian), "The Failure of EU State-Building », contribution présentée à la ASN Convention, New York, 10-12/04/08.

33. Commission européenne, "Stratégie d'élargissement et principaux défis 2006-2007 ", $\operatorname{COM}(2006) 649$ final, Bruxelles, 08/11/06.

34. Conférence de presse d'olli Rehn, 6 février 2008, à l'adresse: [http://ec.europa.eu/ commission_barroso/rehn/pdf/news/080206_or_remarks_serbia_en.pdf]

35. Noutcheva (Gergana), «Fake, partial and imposed compliance: the limits of the EU's normative power in the Western Balkans », European Journal of Public Policy, 16 (7), octobre 2009. 


\section{RÉSUMÉS}

Le présent article interroge le double objectif de l'Union européenne dans les Balkans occidentaux, qui vise à la fois à la stabilisation et à l'association des pays de la région. Double objectif dont découle un double défi: stabiliser cette zone fragile tout en poursuivant l'association des pays à l'UE, encourager la dynamique des réformes sans décrédibiliser le principe de conditionnalité par un assouplissement trop important des exigences prédéfinies. Des études de cas portant sur la Macédoine, la Bosnie-Herzégovine et la Serbie révèlent les difficultés inhérentes à un tel exercice de haute voltige politique.

This article tackles the double objective of the European Union in the Balkans, aiming simultaneously for the stabilisation and the association of the countries of the region. A double objective that results in a double challenge: to stabilise this fragile zone while pursuing the association of the countries to the $\mathrm{EU}$, to encourage the reform dynamic without reducing the credibility of the conditionality principle by an undue softening of the predefined requirements. Case studies on Macedonia, Bosnia-Herzegovina and Serbia demonstrate the difficulties inherent to such a political balancing act.

\section{INDEX}

Index géographique : Balkans, Bosnie-Herzégovine, Europe, Macédoine, Serbie

Mots-clés : Elargissement de l'Union européenne, pacte de stabilisation

\section{AUTEURS}

\section{THERESIA TOEGLHOFER}

Diplômée de Sciences Po Paris en Affaires internationales, spécialisation Management public international ; étudiante en histoire avec spécialisation en Europe du Sud-Est à l'Université de Graz (Autriche).

theresia.toeglhofer@sciences-po.org

\section{NATASHA WUNSCH}

Étudiante en double Master entre Sciences Po Paris (Affaires européennes) et l'Université libre de Berlin (Sciences Politiques); responsable de la rubrique » Balkans » chez Nouvelle Europe. natasha.wunsch@fu-berlin.de 